\title{
Comprensión de los conceptos de perímetro y área en el contexto de la agricultura del café en estudiantes del grado $6^{\circ 1}$
}

\author{
Understanding the concepts of perimeter and area in the \\ context of coffee farming in 6 th grade students
}

Compreender os conceitos de perímetro e área no contexto da cafeicultura nos alunos de 6 a série

Recibido: mayo de 2013

Aprobado: agosto de 2013
Juan David González Molina²

Zaida Margot Santa Ramírez ${ }^{3}$

René Alejandro Londoño Cano ${ }^{4}$

\begin{abstract}
Resumen
La presente investigación se centra en los ambientes locales del aprendiz,
con el fin de darle significado a conceptos geométricos básicos, debido a
que los estudiantes no alcanzan altos niveles de comprensión; prueba de
ello es el uso inapropiado que hacen de los términos matemáticos para
hacer argumentaciones, presentar inquietudes o solicitar explicaciones;
parte de la problemática se debe a la falta de diferenciación de conceptos
clave en el estudio de la geometría, como por ejemplo, área y perímetro.
Así mismo, autores como Corberán (1996) y Chamorro y otros (2008),
detectaron problemas en la comprensión cuando los estudiantes tienen
que relacionar estos dos conceptos.
Resumen
La presente investigación se centra en los ambientes locales del aprendiz,
con el fin de darle significado a conceptos geométricos básicos, debido a
que los estudiantes no alcanzan altos niveles de comprensión; prueba de
ello es el uso inapropiado que hacen de los términos matemáticos para
hacer argumentaciones, presentar inquietudes o solicitar explicaciones;
parte de la problemática se debe a la falta de diferenciación de conceptos
clave en el estudio de la geometría, como por ejemplo, área y perímetro.
Así mismo, autores como Corberán (1996) y Chamorro y otros (2008),
detectaron problemas en la comprensión cuando los estudiantes tienen
que relacionar estos dos conceptos.

Resumen
La presente investigación se centra en los ambientes locales del aprendiz,
con el fin de darle significado a conceptos geométricos básicos, debido a
que los estudiantes no alcanzan altos niveles de comprensión; prueba de
ello es el uso inapropiado que hacen de los términos matemáticos para
hacer argumentaciones, presentar inquietudes o solicitar explicaciones;
parte de la problemática se debe a la falta de diferenciación de conceptos
clave en el estudio de la geometría, como por ejemplo, área y perímetro.
Así mismo, autores como Corberán (1996) y Chamorro y otros (2008),
detectaron problemas en la comprensión cuando los estudiantes tienen
que relacionar estos dos conceptos.

Resumen
La presente investigación se centra en los ambientes locales del aprendiz,
con el fin de darle significado a conceptos geométricos básicos, debido a
que los estudiantes no alcanzan altos niveles de comprensión; prueba de
ello es el uso inapropiado que hacen de los términos matemáticos para
hacer argumentaciones, presentar inquietudes o solicitar explicaciones;
parte de la problemática se debe a la falta de diferenciación de conceptos
clave en el estudio de la geometría, como por ejemplo, área y perímetro.
Así mismo, autores como Corberán (1996) y Chamorro y otros (2008),
detectaron problemas en la comprensión cuando los estudiantes tienen
que relacionar estos dos conceptos.

Resumen
La presente investigación se centra en los ambientes locales del aprendiz,
con el fin de darle significado a conceptos geométricos básicos, debido a
que los estudiantes no alcanzan altos niveles de comprensión; prueba de
ello es el uso inapropiado que hacen de los términos matemáticos para
hacer argumentaciones, presentar inquietudes o solicitar explicaciones;
parte de la problemática se debe a la falta de diferenciación de conceptos
clave en el estudio de la geometría, como por ejemplo, área y perímetro.
Así mismo, autores como Corberán (1996) y Chamorro y otros (2008),
detectaron problemas en la comprensión cuando los estudiantes tienen
que relacionar estos dos conceptos.

Resumen
La presente investigación se centra en los ambientes locales del aprendiz,
con el fin de darle significado a conceptos geométricos básicos, debido a
que los estudiantes no alcanzan altos niveles de comprensión; prueba de
ello es el uso inapropiado que hacen de los términos matemáticos para
hacer argumentaciones, presentar inquietudes o solicitar explicaciones;
parte de la problemática se debe a la falta de diferenciación de conceptos
clave en el estudio de la geometría, como por ejemplo, área y perímetro.
Así mismo, autores como Corberán (1996) y Chamorro y otros (2008),
detectaron problemas en la comprensión cuando los estudiantes tienen
que relacionar estos dos conceptos.

Resumen
La presente investigación se centra en los ambientes locales del aprendiz,
con el fin de darle significado a conceptos geométricos básicos, debido a
que los estudiantes no alcanzan altos niveles de comprensión; prueba de
ello es el uso inapropiado que hacen de los términos matemáticos para
hacer argumentaciones, presentar inquietudes o solicitar explicaciones;
parte de la problemática se debe a la falta de diferenciación de conceptos
clave en el estudio de la geometría, como por ejemplo, área y perímetro.
Así mismo, autores como Corberán (1996) y Chamorro y otros (2008),
detectaron problemas en la comprensión cuando los estudiantes tienen
que relacionar estos dos conceptos.

Resumen
La presente investigación se centra en los ambientes locales del aprendiz,
con el fin de darle significado a conceptos geométricos básicos, debido a
que los estudiantes no alcanzan altos niveles de comprensión; prueba de
ello es el uso inapropiado que hacen de los términos matemáticos para
hacer argumentaciones, presentar inquietudes o solicitar explicaciones;
parte de la problemática se debe a la falta de diferenciación de conceptos
clave en el estudio de la geometría, como por ejemplo, área y perímetro.
Así mismo, autores como Corberán (1996) y Chamorro y otros (2008),
detectaron problemas en la comprensión cuando los estudiantes tienen
que relacionar estos dos conceptos.

Resumen
La presente investigación se centra en los ambientes locales del aprendiz,
con el fin de darle significado a conceptos geométricos básicos, debido a
que los estudiantes no alcanzan altos niveles de comprensión; prueba de
ello es el uso inapropiado que hacen de los términos matemáticos para
hacer argumentaciones, presentar inquietudes o solicitar explicaciones;
parte de la problemática se debe a la falta de diferenciación de conceptos
clave en el estudio de la geometría, como por ejemplo, área y perímetro.
Así mismo, autores como Corberán (1996) y Chamorro y otros (2008),
detectaron problemas en la comprensión cuando los estudiantes tienen
que relacionar estos dos conceptos.

Resumen
La presente investigación se centra en los ambientes locales del aprendiz,
con el fin de darle significado a conceptos geométricos básicos, debido a
que los estudiantes no alcanzan altos niveles de comprensión; prueba de
ello es el uso inapropiado que hacen de los términos matemáticos para
hacer argumentaciones, presentar inquietudes o solicitar explicaciones;
parte de la problemática se debe a la falta de diferenciación de conceptos
clave en el estudio de la geometría, como por ejemplo, área y perímetro.
Así mismo, autores como Corberán (1996) y Chamorro y otros (2008),
detectaron problemas en la comprensión cuando los estudiantes tienen
que relacionar estos dos conceptos.

Resumen
La presente investigación se centra en los ambientes locales del aprendiz,
con el fin de darle significado a conceptos geométricos básicos, debido a
que los estudiantes no alcanzan altos niveles de comprensión; prueba de
ello es el uso inapropiado que hacen de los términos matemáticos para
hacer argumentaciones, presentar inquietudes o solicitar explicaciones;
parte de la problemática se debe a la falta de diferenciación de conceptos
clave en el estudio de la geometría, como por ejemplo, área y perímetro.
Así mismo, autores como Corberán (1996) y Chamorro y otros (2008),
detectaron problemas en la comprensión cuando los estudiantes tienen
que relacionar estos dos conceptos. Palabras clave: Comprensión; matemáticas escolares; geometría; formas
geométricas; perímetro; área; Educación Matemática y otras disciplinas; Palabras clave: Comprensión; matemáticas escolares; geometría; formas
geométricas; perímetro; área; Educación Matemática y otras disciplinas; agricultura del café; enseñanza para la Comprensión.
\end{abstract}

\begin{abstract}
This research focuses on the local environment of the learner, in order to give meaning to basic geometric concepts, because students do not achieve high levels of understanding, the proof is making improper use of mathematical terms to make arguments, raise concerns or ask for explanations, part of the problem is due to the lack of differentiation of key concepts in the study of geometry, such as area and perimeter. Also, authors like Corberán (1996) and Chamorro and others (2008) found problems in

understanding when students have to relate these two concepts.
\end{abstract}

Artículo de Investigación.

Universidad de Antioquia. Medellin, Colombia. Contacto: jdavidgonzalezm@gmail.com

3 Universidad de Antioquia. Medellin, Colombia. Contacto: zsanta@ayura.udea.edu.co

4 Universidad de Antioquia. Medellin, Colombia. Contacto: rene2@une.net.co 
Keywords: Understanding; school mathematics, geometry, geometric shapes, perimeter, area, and other disciplines Mathematics Education, agriculture Coffee; Teaching for Understanding.

\section{Resumo}

Esta investigação centra-se no ambiente local do aluno, a fim de dar significado aos conceitos geométricos básicos, porque os alunos não alcançar altos níveis de entendimento, a prova está fazendo uso indevido de termos matemáticos para fazer argumentos, levantar questões ou pedir explicações, parte do problema se deve à falta de diferenciação de conceitos-chave no estudo da geometria, como área e perímetro. Além disso, autores como Corberán (1996) e Chamorro e outros (2008) encontrou problemas na compreensão quando os alunos têm que relacionar esses dois conceitos.

Palavras-chave: Entendimento; matemática escolar, geometria, formas geométricas, perímetro, área e outras disciplinas Educação Matemática, a agricultura de café; Ensino para a Compreensão.

\section{Presentación del problema}

Dificultades en la comprensión de los conceptos asociados de área y perímetro. En nuestra experiencia docente, hemos identificado que los estudiantes suelen relacionar el área con la aplicación de fórmulas y el desarrollo de algoritmos e, incluso, hemos notado que no dan crédito a que dos figuras de diferente forma y perímetro puedan tener igual área. Algunos autores también han advertido sobre este hecho:

Esta "falsa” relación entre el área y el perímetro, que se ha constatado que está muy arraigada en los alumnos, pone de manifiesto que éstos piensan en el área y en el perímetro como en dos propiedades de la superficie íntimamente ligadas... Algunos investigadores advierten que limitar la enseñanza del área a las fórmulas para su cálculo, se convierte para los alumnos en un obstáculo para comprender el área como número de unidades que recubren la superficie (Corberán, 1996, p. 10).

Considerando el concepto de superficie, Chamorro y otros (2008) aseguran que el proceso de decantación entre longitud y superficie es muy complejo y que es aún más difícil, cuando la primera aproximación a superficie se da con una figura dibujada por el profesor en el tablero, debido a que lo más resaltante para el estudiante es la línea que constituye el límite de la figura plana.

Con relación a la reducción del área a una simple expresión numérica o a un algoritmo de aplicación, sin la debida reflexión sobre el concepto, sus propiedades y la misma estructura de la expresión algebraica, Gómez (1989) cita a Vanlehn (1983) y expone cómo los alumnos generalizan la aplicación de un algoritmo tipo a cualquier expresión así no sean de la misma estructura, por ejemplo: sea la expresión $\mathrm{a}^{\star}(\mathrm{b}+\mathrm{c})=\mathrm{a}^{\star} \mathrm{b}+\mathrm{a}^{\star} \mathrm{c}$, este algoritmo tipo o referente lo aplican a expresiones como $\sqrt{ }$.

La contextualización del aprendizaje. El MEN (1998) hace referencia al contexto, en cuanto a la utilidad que tiene en el aprendizaje de conceptos y en la comprensión de los mismos:

El contexto tiene que ver con los ambientes que rodean al estudiante y que le dan sentido a las matemáticas que aprende. Variables como las condiciones sociales y culturales tanto locales como internacionales, el tipo de interacciones, los intereses que se generan, las creencias, así como las condiciones económicas del ambiente social en el que se concreta el acto educativo, deben tenerse 
en cuenta en el diseño y ejecución de experiencias didácticas (p. 36).

Específicamente, en los Lineamientos Curriculares de Matemáticas se hace referencia al contexto como "elemento importante que puede proveer al individuo de aptitudes, competencias y herramientas para resolver problemas y representar ideas matemáticas..." (MEN, 1998, p. 30).

Planteamiento del problema. El aprendizaje de las matemáticas tiene que ir más allá de la aplicación "en serie" de algoritmos que despejan X, expresan el cuadrado de un binomio, muestran el grado de rotación de una figura o que establecen semejanzas entre triángulos. Las matemáticas, para comprenderse, utilizarse y observarse en las actividades del entorno tienen que ser significadas. Esta significación se expone en sus términos, símbolos, enunciados y formulaciones pero, también, en las interpretaciones que el sujeto quien las estudia da a todos esos elementos; es importante que haya una estrecha relación entre lo que se expresa en las ideas matemáticas y lo que asume quien se adentra en ellas.

Considerando lo anterior, se pretende determinar cómo pueden influir las actividades agrícolas en la comprensión de los conceptos de perímetro y área en los estudiantes, de tal manera que logren una transformación del pensamiento concreto al pensamiento abstracto y que pueda ser un punto de partida para demostrar que algunos contextos de su realidad pueden ser aprovechados para la comprensión de diversos conceptos matemáticos. Por lo tanto, se pretende responder la siguiente pregunta: ¿Cómo comprenden los estudiantes del grado $6^{\circ}$ los conceptos de perímetro y área, estableciendo la independencia de sus medidas, en el contexto de la agricultura del café, en el marco teórico de la Enseñanza para la Comprensión? Para lograrlo, se ha planteado el siguiente objetivo: Analizar el proceso de comprensión de los conceptos de perímetro y área, al establecer la independencia de sus medidas en el contexto de la agricultura del café, en los estudiantes del grado $6^{\circ}$.

\section{Marco conceptual}

Enseñanza para la Comprensión. Este marco conceptual propicia actividades en las que el contexto es utilizado como medio para lograr avances en la comprensión; además, brinda una estructura teórica que define la comprensión, da herramientas para la construcción curricular a través de elementos como los tópicos generativos, metas de comprensión, desempeños y evaluación continua; también, permite la caracterización de la comprensión a través de las dimensiones: contenido, método, propósitos y formas de comunicación y, en cada una de éstas, se determinan los niveles: ingenuo, novato, aprendiz y maestro.

Acevedo (2011), en su trabajo de investigación, presenta la EpC como su marco teórico de referencia. En relación a lo que permite el marco teórico afirma:

Este es un marco que además de dar un aporte teórico sobre la comprensión, brinda a los profesores herramientas para la planificación y diseño de sus prácticas de aula para fomentar la comprensión, desde el abordaje de un concepto hasta el de un curso completo (p. 35).

En los Estándares Curriculares de Matemáticas, con relación al marco teórico, se dice que en este: "se proponen los aspectos relacionados con los métodos y técnicas, con las formas de expresar y comunicar lo comprendido y con la praxis cotidiana, profesional o científico-técnica en que se despliegue dicha comprensión" (MEN, 2006, p. 49).

\section{Metodología}

Por tratarse de la investigación de un fenómeno social que se centra en la interpretación de un proceso de aprendizaje, se abordará un paradigma de corte cualitativo. De hecho, en esta investigación se 
asumen realidad y el conocimiento desde las ideas de Sandoval (2002), quien afirma que:

La realidad necesariamente requiere, para su existencia, de un sujeto cognoscente, el cual está influido por una cultura y unas relaciones sociales particulares, que hacen que la realidad epistémica dependa para su definición, comprensión y análisis, del conocimiento de las formas de percibir, pensar, sentir y actuar propias de esos sujetos cognoscentes... El conocimiento es una creación compartida a partir de la interacción entre el investigador y el investigado, en la cual, los valores median o influyen la generación del conocimiento; lo que hace necesario 'meterse en la realidad' objeto de análisis, para poder comprenderla tanto en su lógica interna como en su especificidad. (p. 28, 29).

Asumiendo que el conocimiento es una construcción social que circula, que necesita de la interacción de sujetos que lo compartan, quelo aprehenden de acuerdo con su individualidad y subjetividad y, que además, "los métodos cualitativos pueden usarse para obtener detalles complejos de algunos fenómenos, tales como sentimientos, procesos de pensamiento y emociones...” (Strauss \& Corbin, 2002, p, 13) se puede justificar que la metodología que orienta la investigación es cualitativa.

\section{Análisis de los datos}

El proceso de análisis de la información se llevará a cabo con el siguiente proceso:

De acuerdo con el marco teórico de la EpC se tienen las siguientes etapas: fase de exploración, fase de investigación guiada, proyecto final de síntesis. Las tres fases, los guiones de entrevistas y los productos esperados están contemplados dentro de lo que en esta investigación se ha denominado Guía Curricular, Ruta de la Comprensión de los Conceptos de Perímetro y Área y la Independencia de sus Medidas.

Es importante considerar que en la investigación cualitativa, los datos obtenidos no están estructurados y, uno de los propósitos del investigador cualitativo, es darles estructura (Hernández, Fernández y Baptista, 2006). Esto requiere las siguientes tareas: transcripción de las entrevistas (grabadas en audio o en video); redacción y relato de las observaciones; codificación, tematización y categorización; todo esto con el fin de desarrollarlos dentro del marco teórico y darles sentido de acuerdo al planteamiento del problema. Todas las acciones y tareas, tienen la finalidad de refinar los descriptores de la comprensión (rúbrica de dimensiones de categorías por nivel) y, a su vez, posibilitar el análisis del proceso de comprensión de los estudiantes.

\section{Posibles conclusiones}

En la etapa tres de la investigación "proyecto final de síntesis", se espera que los estudiantes puedan exponer ante la comunidad educativa lo que aprendieron sobre los conceptos de perímetro y área; en dicha etapa deberán presentar un portafolio, con la evidencia de los trabajos y actividades resueltas; una maqueta a escala de un sembrado de café en la que representen la optimización de un área con un perímetro dado y den a conocer, a través del discurso y ejemplos en diferentes contextos, la independencia de las medidas del perímetro y el área.

En la última exposición se espera que pongan de manifiesto un nivel superior al de novato en cada una de las dimensiones, es decir, que den cuenta del sano escepticismo, del empleo de métodos técnicos, de la vinculación entre diversas áreas del conocimiento y que se expresen de forma fluida y precisa en relación a los objetos de estudio.

\section{Referencias}

Acevedo, D. (2011). Comprensión del Concepto de Probabilidad en Estudiantes de Décimo Grado. Medellín: Universidad de Antioquia.

Chamorro, M.; Belmonte, J.; Llinares, S.; Ruíz, M. \& Vecino Rubio, F. (2008). Didáctica de las Matemáticas para Primaria. España: Pearson Prentice Hall. 
Corberán, R. (1996). El Área, recursos didácticos para su enseñanza en primaria. En O. Mourut, Procesos de transferencia de resultados de investigación de aula: El caso del bajo rendimiento escolar en matemáticas. (págs. 1-87). Distrito Federal: CINVESTAV.

Gómez, C. (1989). La Adquisición del Lenguaje Matemático: un Difícil Equilibrio entre el Rigor y el Significado. Comunicación, Lenguaje y Educación, 3-15.

Hernández, R.; Fernández-Collado, C. \& Baptista Lucio, P. (2006). Metodología de la Investigación (4 ed.). México: Mc Graw Hill.

Ministerio de Educación Nacional. (1998). Lineamientos Curriculares de Matemáticas. Santa Fe de Bogotá D. C.: Delfín Ltda.

Ministerio de Educación Nacional. (2006). Estándares Básicos de Competencias en Lenguaje, Matemáticas, Ciencias y Ciudadanas. Santa Fe de Bogotá D. C.: Imprenta Nacional.

Sandoval, C. (2002). Investigación Cualitativa. Bogotá.: ARFO Editores e Impresos Ltda.
Stone, M. (1999). La Enseñanza para la Comprensión. Vinculación entre la investigación y la práctica. Buenos Aires: Paidós.

características. Tesis de grado. Universidad de Antioquia: Facultad de Educación.

Santa, Z., Jaramillo, C. (2010). Aplicaciones de la geometría del doblado de papel a las secciones cónicas.

Santa. (2011). "Comprensión del concepto de elipse como lugar geométrico". Memorias XIII Conferencia Interamericana de Educación Matemática, CIAEM. Recife (Brasil). Recuperado el 15 de febrero de2012 de http:// www.cimm.ucr.ac.cr/ocs/index.php/xiii_ ciaem/xiii_ciaem/paper/view/2279/996 (s. n.) (s.f.) Revista Virtual Universidad Católica del Norte, (31). Recuperado de: http://revistavirtual.ucn.edu.co/index.php?option=com_contentytask=viewyid=169yItemid $=1$

Urbaneja, P. M. (2001). Divulgamat. Recuperado el 10 de Marzo de 2012 de http://divulgamat. ehu.es/weborriak/Historia/MateOspetsuak/ Inprimaketak/Apolonio.asp 